\title{
MEASUREMENT OF LOCAL EFFECTIVE DIFFUSIVITY IN HETEROGENEOUS BIOFILMS
}

\author{
H. Beyenal*, A. Tanyolaç* and Z. Lewandowski** \\ * Hacettepe University, Chemical Engineering Department, Beytepe 06532, Ankara, \\ Turkey \\ ** Center for Biofilm Engineering, P. O. Box 173980, Room 366 EPS, \\ Montana State University, Bozeman, MT, USA
}

\begin{abstract}
We have developed a novel technique to measure local effective diffusivity distribution in heterogeneous biofilms. Mobile microelectrodes (tip diameter $10 \mu \mathrm{m}$ ) and the limiting current technique were employed to measure the effective diffusivity of electroactive species introduced to natural and artificial biofilms. We calibrated the microelectrodes in artificial biofilms of known effective diffusivity and known density. In mixed population biofilms, local effective diffusivity varied from one location to another and decreased toward the bottom of the biofilm. We related local effective diffusivity to local biofilm density using an empirical equation. Surface-averaged biomass density depended on liquid flow velocity at which the biofilms were grown. The higher the flow velocity, the denser were the biofilms. Our technique permits fast evaluation of local effective diffusivity and biofilm density in heterogeneous biofilms. (c) 1998 Published by Elsevier Science Ltd. All rights reserved
\end{abstract}

\section{KEYWORDS}

Biofilm; density; structure; limiting current; local effective diffusivity; local mass transfer.

\section{INTRODUCTION}

Modeling biofilm activity benefits from the knowledge of substrate diffusivity and biofilm density (Karel $e t$ al. 1985; Fan et al., 1990; Zhang et al., 1995). Three procedures are currently used to determine the average effective diffusivity of substrates in biofilms: (1) using the diffusion-reaction model (Andrews and Tien, 1981), (2) using microsensors and substrate concentration profiles (Lewandowski et al., 1991; Fu et al., 1994) and (3) using the diffusion cell method (Converti et al.,1995). None of those three techniques can evaluate the distribution of mass transfer rate in heterogeneous biofilms, because the mass transfer mechanism is different in cell clusters and in interstitial voids. In the cell clusters, the liquid is stationary and the substrates are transported by molecular diffusion (De Beer et al., 1996). In voids, convection is the major mechanism of mass transport (De Beer et al., 1994; Yang and Lewandowski, 1995).

Many researchers acknowledge the need to study the effective diffusivity in biofilms. Fu et al. (1994) using micro slicing technique found that the effective diffusivity decreased toward the bottom of the biofilm, while the biomass density increased. Their method yielded an average effective diffusivity and an average biomass 
density in slices of biofilms thicker than $100 \mu \mathrm{m}$. Zhang and Bishop (1994a, b) and Zhang et al. (1995) quantified the variations in density, porosity, specific surface area, and mean pore radius for different layers of biofilms using micro slicing technique. They found that effective diffusivity decreased toward the bottom of the biofilm while biomass density increased. Bryers and Drummond (1996), and Lawrence et al. (1991) used scanning confocal laser microscopy (SCLM) with fluorescense recovery after photobleaching (FRAP) to measure diffusivity and mobility of fluorescently labeled materials within biofilms. They concluded that the mechanism of mass transfer in biofilms is not uniform over the entire biofilm but, instead, must be considered a function of biofilm structure. This notion has been corroborated in our laboratory; De Beer $e t$ al. (1997) found that the effective diffusivity of fluorescein, TRITC-IgG and phycoerythrin was different in the cell clusters and in the interstitial voids.

All available results illustrating the variation of local effective diffusivity toward the bottom of the biofilm refer to average values of density and average effective diffusivity in biofilm layers, biofilm voids, or biofilm clusters. The goal of this study was to measure the local effective diffusivity in hydrated biofilms, grown under well-defined hydrodynamic conditions. The local consumption of the ferricyanide introduced to the biofilm reactor was measured by cathodically polarized microelectrodes. The ferricyanide, $\mathrm{Fe}(\mathrm{CN})^{-3}{ }_{6}$, was reduced at the tip of the microelectrode to ferrocyanide, $\mathrm{Fe}(\mathrm{CN})^{-4}{ }_{6}$ and a current was generated in the external circuit according to reaction; $\mathrm{Fe}(\mathrm{CN})^{-3} 6_{6}+\mathrm{e}^{-} \rightarrow \mathrm{Fe}(\mathrm{CN})^{-4}{ }_{6}$. By increasing the polarization potential the current increased until the concentration of $\mathrm{Fe}(\mathrm{CN})^{-3}{ }_{6}$ at the electrode surface reached zero, a condition corresponding to the "limiting current." The limiting current density was calculated by relating the limiting current to the sensing area of the microelectrode.

We related the local limiting current density to the local effective diffusivity by using layers of agar of different and known densities, and of known effective diffusivities of the ferricyanide. The effective diffusivity of the ferricyanide in the agar layers was measured in a diffusion cell. A calibration graph was constructed to relate the limiting current density measured by the microelectrode and the effective diffusivity measured in the diffusion cell. This calibration graph was further used to calculate local effective diffusivity in biofilms from the local limiting current density measurements. Different biofilms were used to identify the effects of growth conditions and type of microorganisms on the local effective diffusivity. Finally, we used an empirical equation reported in the literature to relate local effective diffusivity to the local biomass density.

\section{METHODS}

\section{$\underline{\text { Biofilms }}$}

Reactor. An open channel reactor, made of polycarbonate, $4 \mathrm{~cm}$ deep, $4 \mathrm{~cm}$ wide, and $75 \mathrm{~cm}$ long with a total working volume of $420 \mathrm{~mL}$ was used. The nutrient solution was made of $\mathrm{KH}_{2} \mathrm{PO}_{4}(0.69 \mathrm{mM}), \mathrm{K}_{2} \mathrm{HPO}_{4}$ $(1.5 \mathrm{mM}),\left(\mathrm{NH}_{4}\right)_{2} \mathrm{SO}_{4}(0.079 \mathrm{mM}), \mathrm{MgSO}_{4} .7 \mathrm{H}_{2} \mathrm{O}(0.013 \mathrm{mM})$, and yeast extract $(0.031 \mathrm{~g} / \mathrm{L})$. The inoculum, $1 \mathrm{~mL}$ of frozen stock culture (from the Center for Biofilm Engineering) consisted of: Pseudomonas aeruginosa, Pseudomonas fluorescens, and Klebsiella pneumoniae. The reactor was operated batchwise for $12 \mathrm{~h}$ and then switched to the continuous flow mode for $4-5$ days. Biofilms were grown at two different flow velocities $(1.6 \mathrm{~cm} / \mathrm{s}$ and $3.2 \mathrm{~cm} / \mathrm{s})$. Flow velocity was maintained by recycling the growth medium using peristaltic pumps (Cole-Parmer, Chicago, IL). The nutrient solution was aerated in the mixing chamber. Details of the experimental setup were given by Yang and Lewandowski, (1995). For a comparison, we also grew a pure culture Pseudomonas aeruginosa biofilm at $3.2 \mathrm{~cm} / \mathrm{s}$ flow velocity.

\section{Diffusion cell}

Effective diffusivity of ferricyanide in agar was measured using a diffusion cell consisting of two wellmixed tanks, as described by Converti et al. (1995). Each tank was $11 \mathrm{~cm}$ deep, $10 \mathrm{~cm}$ wide and $10 \mathrm{~cm}$ long, made of polycarbonate and was separated from the other tank by a dialysis membrane (Spectrum ${ }^{\circledR}, 132709$, La Cadena, CA). Agar (BBL 11853 - Bencton Dickinson Microbiology Systems, Cockeysville, MD) was 
prepared in $0.2 \mathrm{M} \mathrm{KCl}$ solution and poured on the dialysis membrane. The entire diffusion cell was immersed in a temperature controlled $\left(25 \pm 1^{\circ} \mathrm{C}\right)$ water bath. During the measurement, ferricyanide diffused from the lower tank to the upper tank through the membrane and through the layer of agar. The concentration of ferricyanide in the upper tank was evaluated spectroscopically at $430 \mathrm{~nm}$ wavelength. The effective diffusivities of ferricyanide for various agar densities were calculated from the solution of flux equations given by Converti et al. (1995).

\section{$\underline{\text { Microelectrodes }}$}

Construction. Microelectrodes for the measurement of the limiting current density were constructed following the procedure described by Yang and Lewandowski (1995). The electrodes with tip diameter 10 $\mu \mathrm{m}$ were used. A voltammetry test was performed for each microelectrode to check their performance in a stagnant electrolyte. Polarization potential was scanned from $-0.2 \mathrm{~V}$ to $-1.2 \mathrm{~V}$ in the solution of ferricyanide. The microelectrodes having a stable limiting current in the voltage range between $-0.6 \mathrm{~V}$ to $-0.9 \mathrm{~V}$ were considered usable. The relation between the limiting current density and the ferricyanide concentration was linear which confirmed that the reaction at the tip of the microelectrode was truly diffusion controlled (Dawson and Trass, 1972).

Limiting current density measurement. Before the measurements of the limiting current density, the nutrient solution in the reactor was slowly drained, making sure that the biofilm remained intact. The remaining nutrients were carefully washed from the reactor with $0.2 \mathrm{M} \mathrm{KCl}$, and replaced with the electrolyte solution made of $0.025 \mathrm{M} \mathrm{K}_{3} \mathrm{Fe}(\mathrm{CN})_{6}$ and $0.2 \mathrm{M} \mathrm{KCl} \mathrm{KCl}$ was used as supporting electrolyte to suppress the electromigration (Gao et al., 1995). This solution was recycled for 2 hours through the reactor before the measurements were taken. The biofilm was prepared in such a way to preserve the structure but it was physiologically inactive (Yang and Lewandowski, 1995). Using microelectrodes we demonstrated that the ferricyanide and the dissolved oxygen were not consumed by the biofilm under these conditions. A commercial calomel electrode (Model 13-620-51, Fisher Scientific, Pittsburgh, PA) was used as the counter/reference electrode. A Hewlett Packard 4140B Multimeter served as a voltage source and picoammeter. The microelectrodes were polarized cathodically to $-0.8 \mathrm{~V}$ against the reference electrode. The limiting current density was calculated from the measured limiting current and the surface area of the microelectrode tip.

Calibration of the effective diffusivity microelectrode. Agar gels of known concentrations (from $2.5 \mathrm{~g} / \mathrm{L}$ to $100 \mathrm{~g} / \mathrm{L}$ ) and known effective diffusivity (measured in the diffusion cell) were used to calibrate the microelectrodes. The agar was dissolved in $0.025 \mathrm{M} \mathrm{K}_{3} \mathrm{Fe}(\mathrm{CN})_{6}$ and $0.2 \mathrm{M} \mathrm{KCl}$ solution. The suspension was slowly heated to $100^{\circ} \mathrm{C}$ until it dissolved, then it was cooled in air to $40^{\circ} \mathrm{C}-60^{\circ} \mathrm{C}$, and then poured as a film (thickness $250 \mu \mathrm{m}-1000 \mu \mathrm{m}$ ) on the bottom of the flat plate reactor without biofilm. The reactor was carefully filled with $0.025 \mathrm{M} \mathrm{K}_{3} \mathrm{Fe}(\mathrm{CN})_{6}$ in $0.2 \mathrm{M} \mathrm{KCl}$ electrolyte solution. The solution was recirculated for at least 2 hours to assure that the ferricyanide equilibrated with the agar. Microelectrodes were mounted on a micromanipulator (Model M3301L, World Precision Instruments, New Haven, CT) equipped with a stepper motor (Model 18503, Oriel, Stratford, CT) controlled by the Oriel Model 20010 interface. The microelectrode was introduced from the top of the reactor perpendicularly to the agar layer. The microelectrode was moved down and the limiting current density was measured at different positions within the agar. The vertical position, $z$, was measured as the distance from the tip of the microelectrode to the bottom of the reactor. Figure 1 shows limiting current density profiles for different effective diffusivities (agar densities). The measured limiting current density was nearly constant within the agar, but suddenly decreased just above the bottom of the reactor, because of the mass transfer limitations near the wall. This effect, also noticed by Yang and Lewandowski (1995), limited application of the microelectrodes to evaluating diffusivities at distances less than $60 \mu \mathrm{m}$ from the bottom for tip diameter between $8 \mu \mathrm{m}$ to 12 $\mu \mathrm{m}$. Consequently, the results of microelectrode measurements at distances less than $60 \mu \mathrm{m}$ from the bottom were rejected. The limiting current density measured by the microelectrodes and the effective diffusivity calculated from the measurements in the diffusion cell gave a linear relation within the limits of tested agar densities: 


$$
\mathrm{D}_{\mathrm{a}}=1.12 \times 10^{-10}+3.69 \times 10^{-12} \mathrm{i}
$$

$D_{\mathrm{a}} \quad=$ Diffusivity of ferricyanide in the agar layer $\left(\mathrm{m}^{2} / \mathrm{s}\right)$

$\mathrm{i} \quad=$ Current density (Ampere $/ \mathrm{m}^{2}$ )

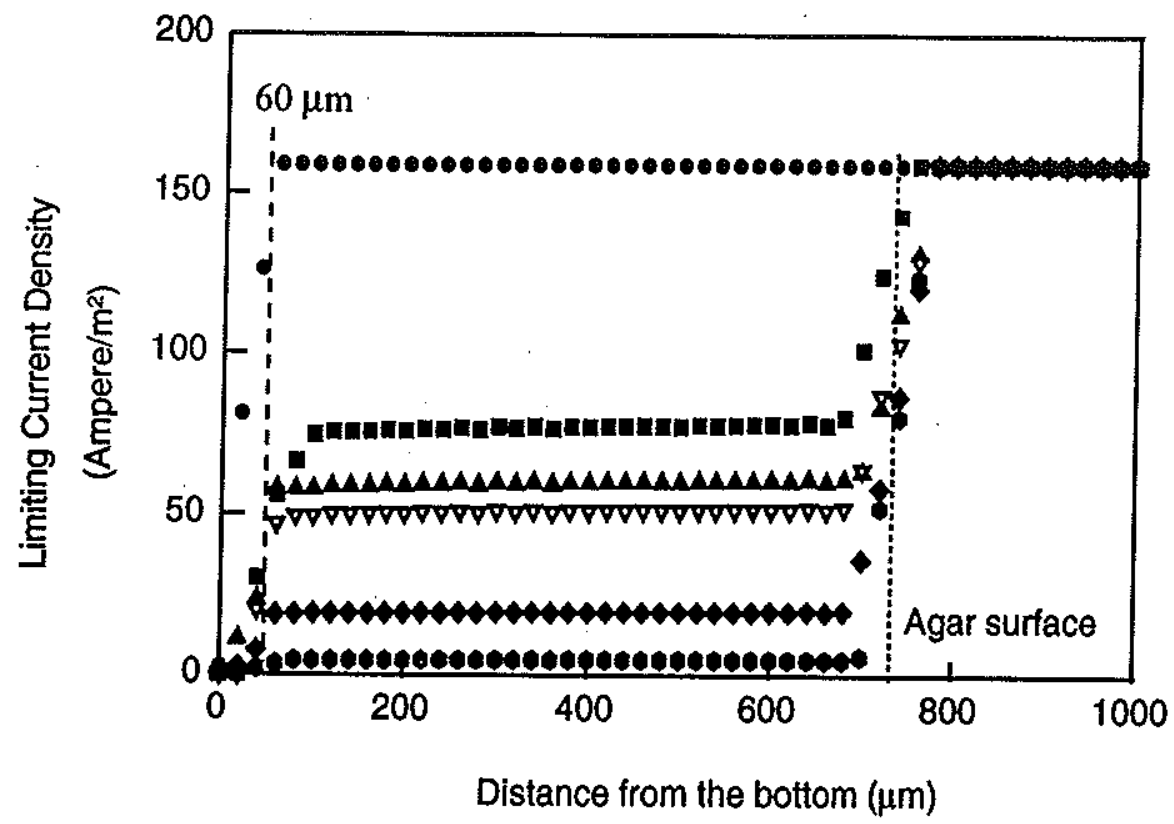

Figure 1. Limiting current density at various distances from the bottom in the electrolyte alone and in agar gels of different densities ( Electrolyte solution ; Electrolyte solution and agar: $\triangle \mathrm{D}_{\mathrm{a}}=3.66 \times 10^{-10} \mathrm{~m}^{2} / \mathrm{s}$; $\Delta$ $\left.D_{a}=3.45 \times 10^{-10} \mathrm{~m}^{2} / \mathrm{s} ; \nabla D_{a}=3.21 \times 10^{-10} \mathrm{~m}^{2} / \mathrm{s} ; \bullet D_{a}=1.98 \times 10^{-10} \mathrm{~m}^{2} / \mathrm{s} ; D_{\mathrm{a}}=1.29 \times 10^{-10} \mathrm{~m}^{2} / \mathrm{s}\right)$.

\section{Measurements in biofilms}

Vertical profiles of limiting current density. The measurements were conducted using the experimental setup described by Yang and Lewandowski (1995). Movements of the microelectrode were computer controlled using Oriel Model 20010 interface. The microelectrode was introduced to the reactor from the top, perpendicularly to the bottom. Position of the electrode tip in the electrolyte solution was monitored using an inverted microscope (Olympus, model CK2, Olympus Optical Co., Ltd. Japan). The microelectrode was positioned above the biofilm and then moved toward the bottom of the reactor at preset increments (usually $20 \mu \mathrm{m})$. The position of the bottom, $\mathrm{z}=0$, was used as a reference and the vertical positions of the microelectrode, $z$, were then determined as the distance between the tip of the microelectrode and the bottom of the reactor. Custom software was used to control the microelectrode movement and to monitor the limiting current. At each position of the microelectrode the data acquisition system (Model CIODAS08PGL, Computer Boards, Inc., Mansfield, MA) collected 22 separate readings at a sampling frequency of $1 \mathrm{kHz}$. The highest and the lowest values were trimmed and the remaining 20 measurements were averaged. The coefficient of variation (CV) was calculated for 20 measurements and compared with the preset acceptable $\mathrm{CV}$. If the measured $\mathrm{CV}$ was smaller than that preset, the measurement was accepted, the data stored, and the microelectrode moved to the next position. If the calculated $\mathrm{CV}$ was higher than that preset, the measurement was repeated. A relative CV of $5 \%$ was used as an arbitrary, acceptable level for all measurements. The profile of limiting current versus depth was displayed on a computer monitor in real time.

Horizontal distribution of limiting current density. Limiting current density was mapped at successive vertical distances from the bottom to evaluate the distribution of the effective diffusivity in the biofilm. The biofilm reactor was positioned on an X-Y micropositioner stage (Model CTC-462-2S, Micro Kinetics, Laguna Hills, CA) with the microelectrode attached to a linear micropositioner (Model CTC-322-20, Micro Kinetics) and nositioned above the reactor. Both micropositioners were comnuter controlled through a 
controller (CTC-283-3, Micro Kinetics), with positioning precision of $0.1 \mu \mathrm{m}$. Custom software was used to simultaneously control the stage movement, the microelectrode movement, and the data acquisition. For the measurements, $10 \times 10$ grid matrix with a step size of $25 \mu \mathrm{m}$ in both $\mathrm{X}$ and $\mathrm{Y}$ directions was used. The data acquisition protocol was the same as that used to measure the vertical profiles of the limiting current density.

The microelectrode was manually positioned above the first grid point. The system was started, the microelectrode was moved into the biofilm to the predetermined distance from the bottom. After the limiting current was measured and the data accepted, the linear micropositioner moved the microelectrode out of the biofilm into the bulk liquid, the motorized $\mathrm{X}-\mathrm{Y}$ stage moved the reactor to the next grid point, and the microelectrode was lowered to the predetermined position at the same distance from the bottom as for the previous measurement. Limiting current densities measured at the same distance from the bottom formed a contour map. The map was displayed on the computer's monitor in real time. The calculated local effective diffusivities at the grid points were arithmetically averaged over the surface at each level in biofilm.

\section{RESULTS AND DISCUSSION}

\section{Distribution of local effective diffusivity}

Profiles of local effective diffusivity in mixed population biofilms grown at a flow velocity $1.6 \mathrm{~cm} / \mathrm{s}$ and in the pure culture of $P$ seudomonas aeruginosa biofilm grown at a flow velocity $3.2 \mathrm{~cm} / \mathrm{s}$ are shown in Figure 2. For the mixed population biofilm, local effective diffusivity profiles oscillated with distance, showing the lowest values of local effective diffusivity, $1.8 \times 10^{-10} \mathrm{~m}^{2} / \mathrm{s}$, near the bottom of the biofilm. The local diffusivity increased toward the bulk-biofilm interface reaching a local maximum $3.2 \times 10^{-10} \mathrm{~m}^{2} / \mathrm{s}, 300 \mu \mathrm{m}$ above the bottom. It then rapidly decreased to $1.5 \times 10^{-10} \mathrm{~m}^{2} / \mathrm{s}$ at $400 \mu \mathrm{m}$ from the bottom, only to increase again $500 \mu \mathrm{m}$ above the bottom. The highest value of the effective diffusivity, $4.4 \times 10^{-10} \mathrm{~m}^{2} / \mathrm{s}$, was measured near the biofilm surface. The profile of local effective diffusivity measured in the pure culture biofilm cluster of Pseudomonas aeruginosa did not show such fluctuations. However, the thickness of the Pseudomonas aeruginosa biofilm was only $140 \mu \mathrm{m}$, while the mixed population biofilm was $500 \mu \mathrm{m}$.

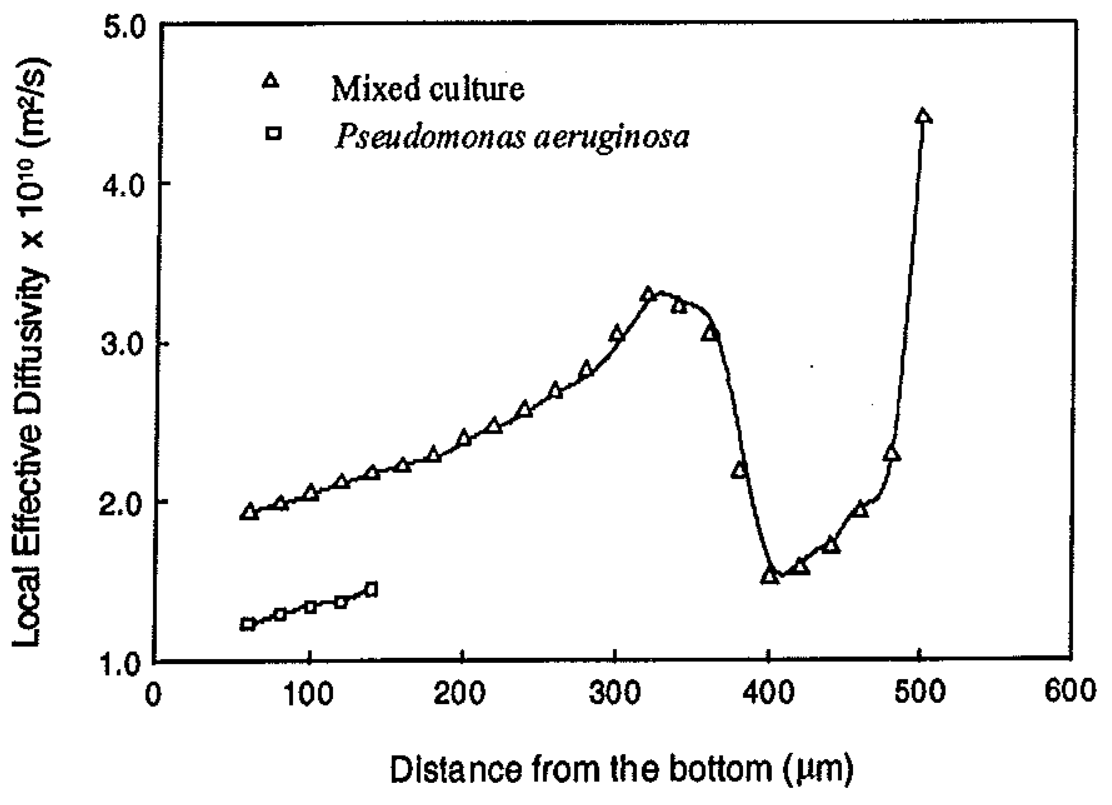

Figure 2. Profiles of local effective diffusivity.

Profiles of limiting current density were measured under stagnant conditions. Effective diffusivity in mixed population biofilms fluctuated with depth into the biofilm (Figure 2), possibly because of the presence of microcolonies separated by interstitial voids. According to our previous studies using confocal scanning laser microscope such microcolonies and voids in mixed population biofilms can be expected. When a pure 
culture biofilm of Pseudomonas aeruginosa was used, effective diffusivity profiles did not show such fluctuations. However, this biofilm was only $140 \mu \mathrm{m}$ thick. Van Loosdrecht (1995) indicated an interesting possibility that biomass density variation in biofilms may be associated with distribution of physiological groups of organisms, producing different polymers. Since the effective diffusivity in biofilms is controlled by the biofilm density (Fan et al., 1990), such biofilms would show fluctuations in effective diffusivity profiles.

\section{Surface averaged effective diffusivity profiles}

Surface averaged effective diffusivities at different levels in biofilms are shown in Figure 3. We did not show the data near the bottom of the biofilm because in this region our microelectrodes were not reliable due to the mass transfer limitations near the reactor wall (see Figure 1). Surface averaged effective diffusivities increased toward the biofilm-bulk liquid interface (Figure 3). This effect may be related to an increase in biofilm porosity -decrease in biofilm density- toward the surface (Zhang and Bishop, 1994a).

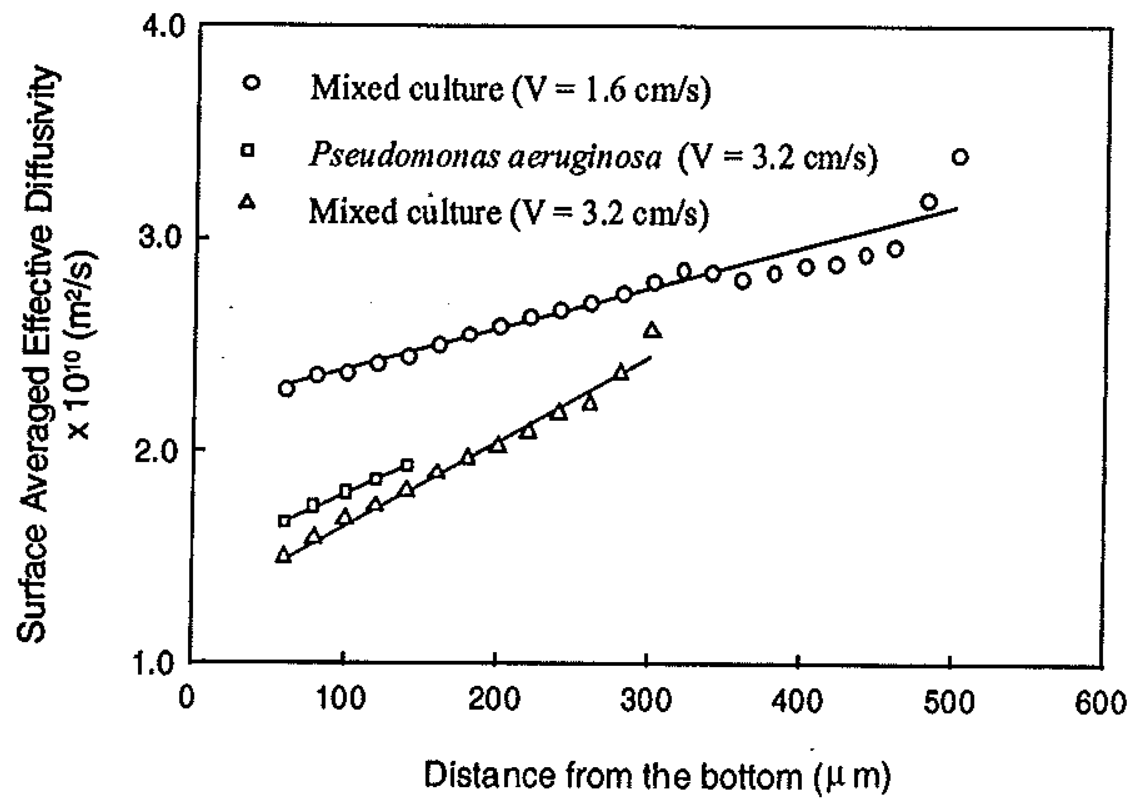

Figure 3. Profiles of surface averaged effective diffusivity in biofilms.

We were not able to directly measure the actual local biomass density in the biofilm. However, Fan et al. (1990) suggested, based on extensive literature studies of effective diffusivity measured in biofilms, activated sludge flocs, and mycelial pellets, the following empirical relation between average biofilm density and average effective diffusivity.

$$
D_{f}=D\left[1-\frac{0.43 X_{f}^{0.92}}{\left(11.19+0.27 X_{f}^{0.99}\right.}\right]
$$

D = Molecular diffusivity of reacting species in the electrolyte or medium $\left(\mathrm{m}^{2} / \mathrm{s}\right)$

$D_{\mathrm{f}} \quad=$ Effective diffusivity of ferricyanide in the biofilm $\left(\mathrm{m}^{2} / \mathrm{s}\right)$

$\mathrm{X}_{\mathrm{f}} \quad=$ Average biofilm density $\left(\mathrm{kg} / \mathrm{m}^{3}\right)$

Using our results from microelectrode measurements (Figure 3) and Equation 2 we estimated the biofilm density profiles in our biofilms (Figure 4). In the mixed population biofilm grown at a flow velocity 1.6 $\mathrm{cm} / \mathrm{s}$, the highest biomass density $55 \mathrm{~g} / \mathrm{L}$ was near the bottom of the biofilm and the biomass density decreased linearly toward the biofilm-liquid interface at a rate of $0.042 \mathrm{~g} / \mathrm{L} / \mu \mathrm{m}$. For mixed population biofilms grown at a flow velocity of $3.2 \mathrm{~cm} / \mathrm{s}$, the biomass density near the bottom of the biofilm was $90 \mathrm{~g} / \mathrm{L}$ 
and it decreased linearly toward the biofilm-liquid interface at a rate of $0.180 \mathrm{~g} / \mathrm{L} / \mu \mathrm{m}$. For pure culture of Pseudomonas aeruginosa biofilms grown at a flow rate of $3.2 \mathrm{~cm} / \mathrm{s}$, the biomass density near the bottom of the biofilm was $80 \mathrm{~g} / \mathrm{L}$ and it again decreased linearly toward the biofilm-liquid interface at the same rate as in the mixed population biofilms, $0.180 \mathrm{~g} / \mathrm{L} / \mu \mathrm{m}$. Increasing flow rate over the propagating biofilm increased the average biofilm density and its gradient through the biofilm regardless of culture homogeneity. This increase is possibly due to the close biomass arrangement in the biofilm and/or secretion of extra cellular polymer to reinforce the structure against overwhelming shear stress on the biofilm. Since maximum shearing moment applied by flow on the biofilm structure appears near the bottom, one can anticipate the maximum robustness at the biofilm-support interface.

The trends in our results correspond well to those already published. Zhang and Bishop (1994a) reported that, for their biofilms, the ratio of effective diffusivity in biofilm to the bulk solution diffusivity ( $\left.D_{f} / D\right)$ decreased from $0.68-0.81$ in the top layer to $0.38-0.45$ in the bottom layer. In our study, $\left(D_{f} / D\right)$ decreased from 0.49 in the top layer to 0.25 in the bottom layer for mixed population biofilm grown at a flow rate of $1.6 \mathrm{~cm} / \mathrm{s}$. The $\left(D_{f} / D\right)$ ratio in mixed population biofilms grown at $3.2 \mathrm{~cm} / \mathrm{s}$ flow velocity decreased from 0.37 in the top layer to 0.16 in the bottom layer. When a pure culture of Pseudomonas aeruginosa biofilm was used, the ratio $\left(D_{f} / D\right)$ decreased from 0.27 in the top layer to 0.21 in the bottom layer. This biofilm, however, was only $140 \mu \mathrm{m}$ thick.

Using micro-slicing techniques, Zhang et al. (1995) and Zhang and Bishop (1994a) measured the highest biomass density, $120 \mathrm{~g} / \mathrm{L}$, near the bottom of the biofilm. The lowest density was measured near the top layers of biofilm $20 \mathrm{~g} / \mathrm{L}-30 \mathrm{~g} / \mathrm{L}$ which agrees well with our study (Figure 4). The advantage of our technique over those employed by other authors rests in its simplicity and the high spatial resolution.

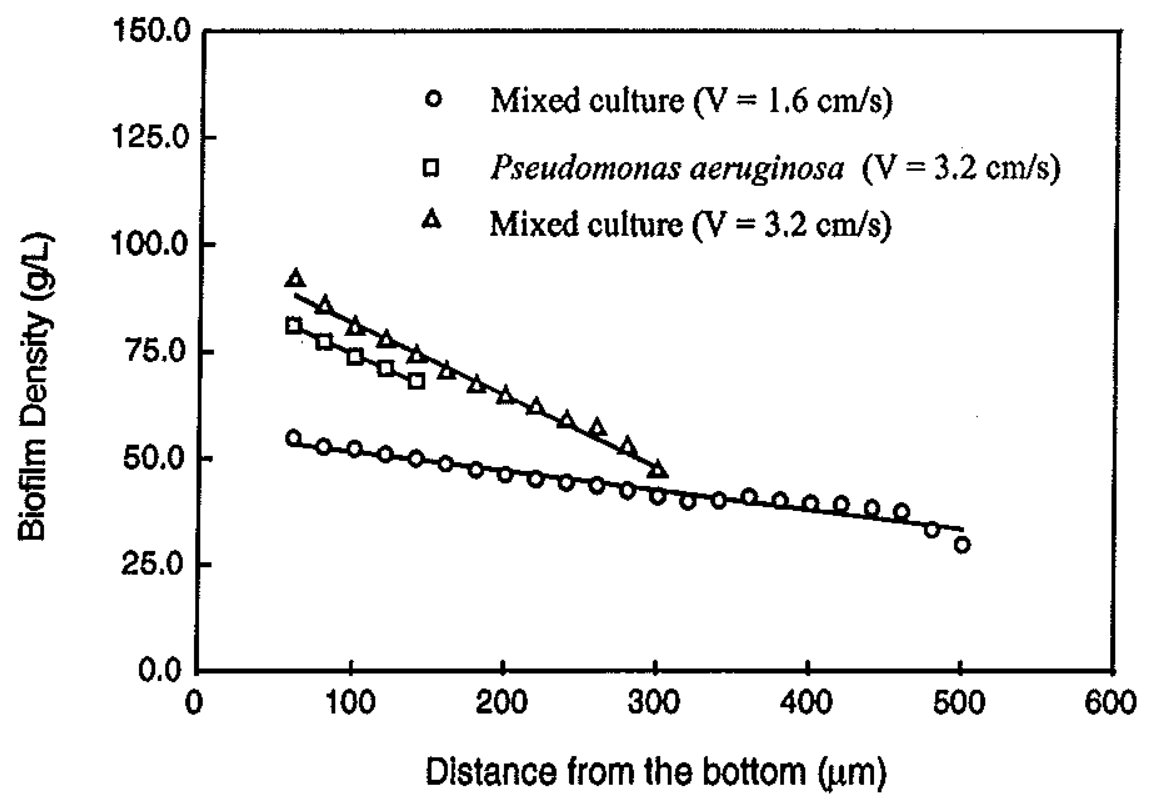

Figure 4. Profiles of average biofilm density in biofilms.

\section{CONCLUSIONS}

A novel technique has been developed to measure the local effective diffusivity distribution in heterogeneous biofilms and successfully applied to a biofilm system. Effective diffusivity in biofilms varied in three dimensions and decreased toward the bottom of a biofilm. Vertical profiles of effective diffusivity in mixed population biofilms showed significant fluctuations, possibly due to the presence of microcolonies separated by interstitial voids or due to the spatial distribution of physiological groups of microorganisms producing different polymers. However, local effective diffusivity in a pure culture biofilm of Pseudomonas auruginosa did not show significant fluctuations. The average flow velocity during biofilm growth affected 
the local effective diffusivity profile of the biofilm. Pure and mixed culture grown at the same flow rate gave the same variation of local effective diffusivity with thickness.

\section{ACKNOWLEDGMENTS}

The research was supported by the cooperative agreement EED-8907039 between the National Science Foundation and Montana State University. Haluk Beyenal was supported by NATO-A2 PhD fellowship program of the Turkish Scientific and Technical Research Council. The authors thank Martin Hamilton and Frank Roe for their valuable advise and discussions.

\section{REFERENCES}

Andrews, G. F. and Tien, C. (1981). Bacterial film growth in adsorbent surfaces. $A I C h E J ., 27,396-403$.

Bryers, D. J. and Drummond, F. (1996). Local mass transfer coefficients in bacterial biofilms using fluorescence recovery after photobleaching (FRAP). In: Progress in biotechnology 11, Immobilized cell: Basics and Applications, R. H. Wijffels, R. M. Buitelaar, C. Bucke, and J. Tramper (eds), Elsevier, New York, pp. 196-204.

Converti, A., Casagrande, M., De Giovanni, M., Rovatti, M. and Del Borghi, M. (1995). Evaluation of glucose diffusion coefficient through cell layers for the kinetic of an immobilized cell bioreactor. Chem. Engng. Sci., 51, 1023-2026.

Dawson, A. D. and Trass, O. (1972). Mass transfer at rough surfaces. J. Heat Mass Transfer, 15, 1317-1336.

De Beer, D., Stoodley, P. and Lewandowski, Z. (1994). Liquid flow in heterogeneous biofilms. Biotechnol. Bioeng., 44, 636-641.

De Beer, D., Stoodley, P. and Lewandowski, Z. (1996). Liquid flow and mass transport in heterogeneous biofilms. Wat. Res., 30, 2761-2765.

De Beer, D., Stoodley, P. and Lewandowski, Z. (1997). Measurement of local diffusion coefficients in biofilms by microinjection and confocal microscopy. Biotechnol. Bioeng., 53, 151-158.

Fan, L.-S., Ramos, R. L., Wisecarver, K. D. and Zehner, B. J. (1990). Diffusion of phenol through a biofilm grown on activated carbon particles in a draft-tube three-phase fluidized bed bioreactor. Biotechnol. Bioeng., 35, 279-286.

Fu, Y. -C., Zhang, T. C. and Bishop, P. L. (1994). Determination of effective oxygen diffusivity in biofilms grown in a completely mixed biodrum reactor. Wat. Sci. Tech., 29(10), 455-462.

Gao, X., Lee, J. and White, H. S. (1995). Natural convection at microelectrodes. Anal. Chem., 67, 1541-1545.

Karel, S. F., Libicki, S. B. and Robertson, C. R. (1985). The immobilization of whole cells: Engineering principles. Chem. Engng. Sci., 40, 1321-1354.

Lawrence, J. R., Korber, D. R., Hoyle, B. D., Costerton, J. W. and Caldwell, D. E. (1991). Optical sectioning of microbial biofilms. J. Bacteriol., 173, 6558-6567.

Lewandowski, Z., Walser, G. and Characklis, W. G. (1991). Reaction kinetics in biofilms. Biotechnol. Bioeng., 38, $877-882$.

van Loosdrecht, M. C. M., Eikelboom, D., Gjaltema, A., Mulder, A., Thijhus, L., and Heijnen, J. J. (1995). Biofilm structures. Wat. Sci. Tech, 32 (8), 35-43.

Yang, S. and Lewandowski, Z. (1995). Measurement of local mass transfer coefficient in biofilms. Biotechnol. Bioeng., 48, 737744.

Zhang, T. C. and Bishop, P. L. (1994a). Evaluation of tortuosity factors and effective diffusivities in biofilms. Wat. Res., 28, 22792287.

Zhang, T. C. and Bishop, P. L. (1994b). Density, porosity, and pore structure of biofilms, Wat.Res., 28, 2267-2277.

Zhang, T. C., Fu, Y. C., Bishop, P. L., Kupferle, M., FitzGerald, S., Jiang, H. H. and Harmer, C. (1995). Transport and biodegradation of toxic organics in biofilms. J. Hazardous Materials., 41: 267-285. 